\title{
Interplay between size and stability of magnetic skyrmions
}

\author{
A. S. Varentsova ${ }^{1}$, M. N. Potkina ${ }^{2,3}$, S. von Malottki ${ }^{4}$, S. Heinze ${ }^{4}$, P. F. Bessarab ${ }^{1}$ \\ ${ }^{1}$ ITMO University, 197101 St. Petersburg, Russia \\ ${ }^{2}$ St. Petersburg State University, 198504 St. Petersburg, Russia \\ ${ }^{3}$ Science Institute, University of Iceland, 107 Reykjavík, Iceland \\ ${ }^{4}$ Institute of Theoretical Physics and Astrophysics, University of Kiel, 24098 Kiel, Germany \\ varentsova.a@mail.ru, bessarab@hi.is
}

PACS 75.70.Ak, 75.70.Kw

DOI 10.17586/2220-8054-2018-9-3-356-363

\begin{abstract}
The relationship between the size and stability of isolated skyrmions in a magnetic monolayer is analyzed based on minimum energy path calculations and atomistic spin Hamiltonian. It is demonstrated that the energy barrier protecting the skyrmion from collapse to the ferromagnetic state is not uniquely defined by the skyrmion size, although these two properties as functions of relevant material parameters follow similar trends. Stability of nanoscale skyrmions can be enhanced by a concerted adjustment of material parameters. The proposed parameter transformation conserves the skyrmion size, but does not conserve the skyrmion shape which changes from an arrow-like pattern to a profile that resembles magnetic bubbles. This transformation of the skyrmion shape is accompanied by an increase in the collapse energy barrier and thus enhancement of skyrmion stability.
\end{abstract}

Keywords: magnetic skyrmion, thermal stability, energy barrier.

Received: 1 May 2018

Revised: 10 May 2018

\section{Introduction}

The field of localized, topological spin textures such as magnetic skyrmions [1-3] is currently receiving a lot of attention. The interest in skyrmions is motivated by their unique properties potentially making them perfect information carriers [4-7]. Sensitivity of these spin swirls to pulses of electric current [8-10] benefits the efficient control of information flows and facilitates qualitative decrease in power consumption of data processing devices. The small size of skyrmions could increase data storage density far beyond what state-of-the-art technology offers today. For nanoscale skyrmions, thermal stability becomes an issue as thermal fluctuations can spontaneously destroy skyrmion states and, therefore, corrupt the stored data. Available experimental data on magnetic skyrmions in various materials and material combinations demonstrates inverse relationship between the skyrmion size and skyrmion stability: small skyrmions tend to be less stable compared to large ones [8,11-21]. For example, roomtemperature skyrmions in a $\mathrm{Pt} / \mathrm{CoFeB} / \mathrm{MgO}$ heterostructure are roughly $100 \mathrm{~nm}$ in diameter [18], which is more than an order of magnitude larger than skyrmions observed in a $\mathrm{Pd} / \mathrm{Fe} / \mathrm{Ir}(111)$ system only at low temperatures by means of spin-polarized scanning tunneling microscopy $[19,20]$. The question then arises as to how general this relation between skyrmion size and skyrmion stability is and whether it is possible to design an optimal medium where magnetic skyrmions are small enough while being sufficiently stable at ambient conditions.

Equilibrium properties of skyrmions such as size and shape have intensively been studied since the very discovery of magnetic skyrmions more than twenty years ago [2-4,22-26], but quantitative characterization of skyrmion stability has started being addressed rather recently [27-38]. Thermal stability can be quantified by calculating skyrmion lifetime using harmonic transition state theory for spins [39]. The theory predicts an Arrhenius expression for the skyrmion lifetime $\tau$ as a function of temperature $T$,

$$
\tau=\tau_{0} e^{\Delta E / k_{B} T}
$$

where the magnitude of both the energy barrier $\Delta E$ and the pre-exponential factor $\tau_{0}$ depends on the mechanism of skyrmion annihilation [38]. Theoretical calculations applied to atomistic spin Hamiltonian have previously revealed two mechanisms of skyrmion decay into a polarized, ferromagnetic configuration: Radial collapse of a skyrmion [27,31] and escape of the skyrmion through the system boundary [32-34]. Relative contributions of these mechanisms to the skyrmion stability have been studied as a function of relevant material parameters [32], applied magnetic field [35,38] and presence of impurities [33,36]. The effect of long-range, frustrated exchange interaction on skyrmion stability has also been addressed using the spin Hamiltonian parametrized from density functional theory calculations [37]. It has been shown that taking exchange beyond the nearest neighbors into 
account results in significant enhancement of energy barriers for the skyrmion collapse compared to what effective, nearest-neighbor models predict [37]. Overall, significant amount of knowledge on skyrmion size and skyrmion stability has been accumulated. However, these two properties have so far never been directly linked. Careful analysis of the interplay between skyrmion size and stability is of critical importance for the use of skyrmions in future information technologies.

In this article, the relationship between the size and stability of an isolated Néel skyrmion is explored using minimum energy path calculations and a two-dimensional Heisenberg exchange model. By tracing contours of constant size and collapse energy barrier on a magnetic phase diagram, it is demonstrated that there is no one-toone correspondence between the skyrmion size and skyrmion stability, although these two properties as functions of relevant material parameters follow similar trends. The energy barrier for the skyrmion collapse into the ferromagnetic background is not a unique function of the skyrmion size, which makes it possible to adjust stability of nanoscale skyrmions. In particular, it is shown using the constant pre-exponential factor approximation that the lifetime of fixed-size skyrmions can be enhanced by several orders of magnitude by concerted modification of Dzyaloshinskii-Moriya (DM) interaction and out-of-plane magnetocrystalline anisotropy.

This article is organized as follows. In Section 2, theoretical background and details of numerical simulations are described. In Section 3, parameter dependence of an isolated skyrmion size and stability is summarized, zerotemperature stability diagram equipped with contours of constant skyrmion diameter and collapse energy barrier is obtained, and stability variation of constant-size skyrmions is quantified. Summary and outlook are presented in Section 4 .

\section{Methods}

A two-dimensional skyrmionic system is modeled as a single monolayer of classical spins on a hexagonal lattice. The Hamiltonian of the system includes Heisenberg exchange coupling, antisymmetric DM interaction, and out-of-plane anisotropy. The energy of the system is given by the following equation:

$$
E=-\frac{J}{2} \sum_{\langle i, j\rangle} \vec{m}_{i} \cdot \vec{m}_{j}-\frac{D}{2} \sum_{\langle i, j\rangle} \vec{d}_{i j} \cdot\left[\vec{m}_{i} \times \vec{m}_{j}\right]-K \sum_{i}\left(\vec{m}_{i} \cdot \vec{e}_{K}\right)^{2} .
$$

Here, the angular brackets indicate summation over nearest neighbors only, $\vec{m}_{i}$ is the unit vector defining the orientation of the magnetic moment at site $i, J$ and $D$ are the exchange and DM interaction parameters, respectively, $K$ is the anisotropy constant. The unit DM vector $\vec{d}_{i j}$ lies in the monolayer plane and points perpendicular to the bond connecting sites $i$ and $j$. The unit vector $\vec{e}_{K}$ defining the easy axis is normal to the monolayer plane. The Zeeman term is not included in the calculations, so as to mimic technologically relevant ambient conditions with zero applied magnetic field. Magnetostatic dipole-dipole interactions are included effectively in the anisotropy energy [3,31,40]. Equation (2) defines a multidimensional energy surface as a function of orientation of magnetic vectors localized on the lattice sites. Over a certain range of material parameter values, Néel-type skyrmions emerge as local energy minima. Properties of isolated skyrmions are of interest here. Therefore, only one single skyrmion is placed in the simulated system. The size of the computational domain is chosen to be large enough for the isolated, equilibrium skyrmion solution not to be affected by the boundaries. Periodic boundary conditions are applied so as to model extended two-dimensional systems.

For a given value of material parameters $J, D$ and $K$, an isolated skyrmion solution is obtained by taking some rough initial guess for the skyrmion profile and then bringing that to a local minimum on the energy landscape using the velocity projection optimization method [27]. Relaxed, energy-minimum skyrmion configuration is then fitted by a two-parameter profile [20]

$$
\Theta(c, w ; r)=\pi+\sum_{\alpha= \pm 1} \arcsin \left[\tanh \left(\frac{-r+\alpha c}{w / 2}\right)\right],
$$

where $\Theta(c, w ; r)$ is the polar angle of the spin located at the distance $r$ from the skyrmion center and $c, w$ are the fit parameters. Based on the obtained profile, the skyrmion radius $R$ is evaluated using the definition of Bogdanov and Hubert [3]:

$$
R=r_{0}-\Theta_{0}\left(\frac{d \Theta}{d r}\right)_{0}^{-1}
$$

where $\left(r_{0}, \Theta_{0}\right)$ is the steepest slope point of the profile $\Theta(c, w ; r)$.

Skyrmion stability is quantified using minimum energy path (MEP) calculations applied to the skyrmion collapse into the ferromagnetic (FM) state. The highest maximum along the MEP corresponds to the first order saddle point (SP) of the energy surface representing the bottleneck of the skyrmion decay. Within the harmonic 
transition state theory (HTST), the SP energy relative to the skyrmion energy minimum gives the energy barrier $\Delta E$ [39] which defines the lifetime, see Eq. (1). A Geodesic Nudged Elastic Band (GNEB) method [27,41] is used to find MEPs of skyrmion annihilation. The GNEB method involves taking the initial guess of the path represented by a discrete chain of states of the system, and systematically bringing that to the MEP by zeroing the transverse component of the gradient force at each point along the path. In order to distribute the states evenly along the path, virtual springs are introduced between adjacent states. At each state, a local tangent to the path is estimated and the GNEB force guiding the states towards the MEP is defined as the sum of the transverse component of the negative energy gradient and the component of the spring force along the tangent. The positions of states are then adjusted using some optimization algorithm, e.g. velocity projection optimization [27], so as to zero the GNEB force. In this method, both the GNEB force and the path tangent are defined in the local tangent space to the curved configuration space, which is needed to satisfy constraints on the length of magnetic moments and to properly decouple the perpendicular component of the energy gradient from the spring force. The shortest path connecting the skyrmion state and the FM state is taken to be the initial guess for the GNEB calculations. The MEPs obtained with such initial guess correspond to the radial collapse of the skyrmion. Radial collapse is likely the only mechanism of skyrmion annihilation in extended two-dimensional systems described by the Hamiltonian in Eq. (2) $[29,31,41]$ and the only one considered here.

\section{Results}

Within a certain material parameter range, the atomistic model represented by Eq. (2) and its micromagnetic counterpart predict isolated skyrmion solutions as local minima of the energy functional $[3,4,25]$. Analysis involving both numerical calculations and asymptotic methods have previously revealed basic properties of isolated skyrmion solutions. In particular, it has been shown that the equilibrium size of an isolated skyrmion decreases with the anisotropy parameter but increases as the DM interaction strength increases [2,3,25,43]. This behavior is consistent with analytical solutions based on ansatzes such as the one in Eq. (3) [4,25,44]. A representative dependence of the skyrmion diameter on $K / J$ for two fixed values of $D / J$ is obtained using the model given by Eq. (2) and shown in Fig. 1(a). The curves are characterized by a certain critical value of the anisotropy parameter, at which the skyrmion size diverges. Observe that confined skyrmions can demonstrate opposite trends for the skyrmion size compared to the isolated skyrmions considered here $[2,42,43,45]$.
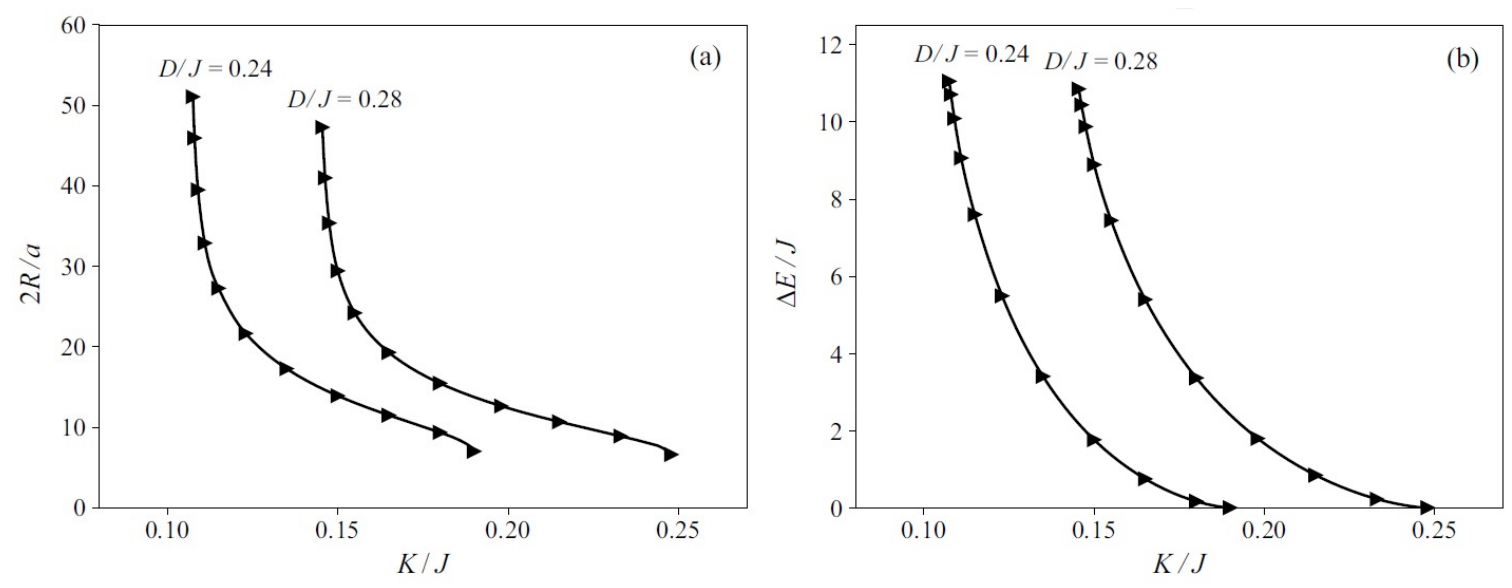

FIG. 1. (a) Skyrmion diameter in units of the lattice constant $a$, and (b) collapse energy barrier in units of the exchange interaction constant $J$ as functions of the anisotropy parameter for two distinct DM interaction strengths. Triangles indicate the data points. Solid lines show the result of cubic spline interpolation between the points

Figure 1(b) shows results of GNEB calculations of the energy barrier $\Delta E$ for the skyrmion collapse into the FM background. With increasing anisotropy strength, energy barrier decreases monotonically to zero. Larger DM interaction parameter results in a larger barrier. It has also been demonstrated in previous calculations that the barrier decreases as the magnetic exchange parameter $J$ gets larger [32]. Material parameter dependence of the collapse energy barrier thus follows the trends for the skyrmion size, as was pointed out by Stosic et al. [32]. This behavior is consistent with an intuitive interpretation of the skyrmion annihilation via radial collapse: The corresponding energy barrier should increase with the number of core spins that need to be flipped into the FM 
state during the skyrmion collapse process and, therefore, should get larger as the skyrmion size increases. It is demonstrated below that this interpretation is actually incomplete and the collapse barrier is not uniquely defined by the skyrmion size. The barrier can be adjusted by a concerted parameter transformation preserving the skyrmion size. A trivial solution is to scale all material parameters - J, D and $K$ - by the same factor, but such an approach gives only a limited control of the skyrmion stability. Below, the distribution of the collapse energy barriers and skyrmion diameters is superimposed on the magnetic phase diagram with the purpose of gaining more insight into the relationship between the skyrmion size and skyrmion stability.
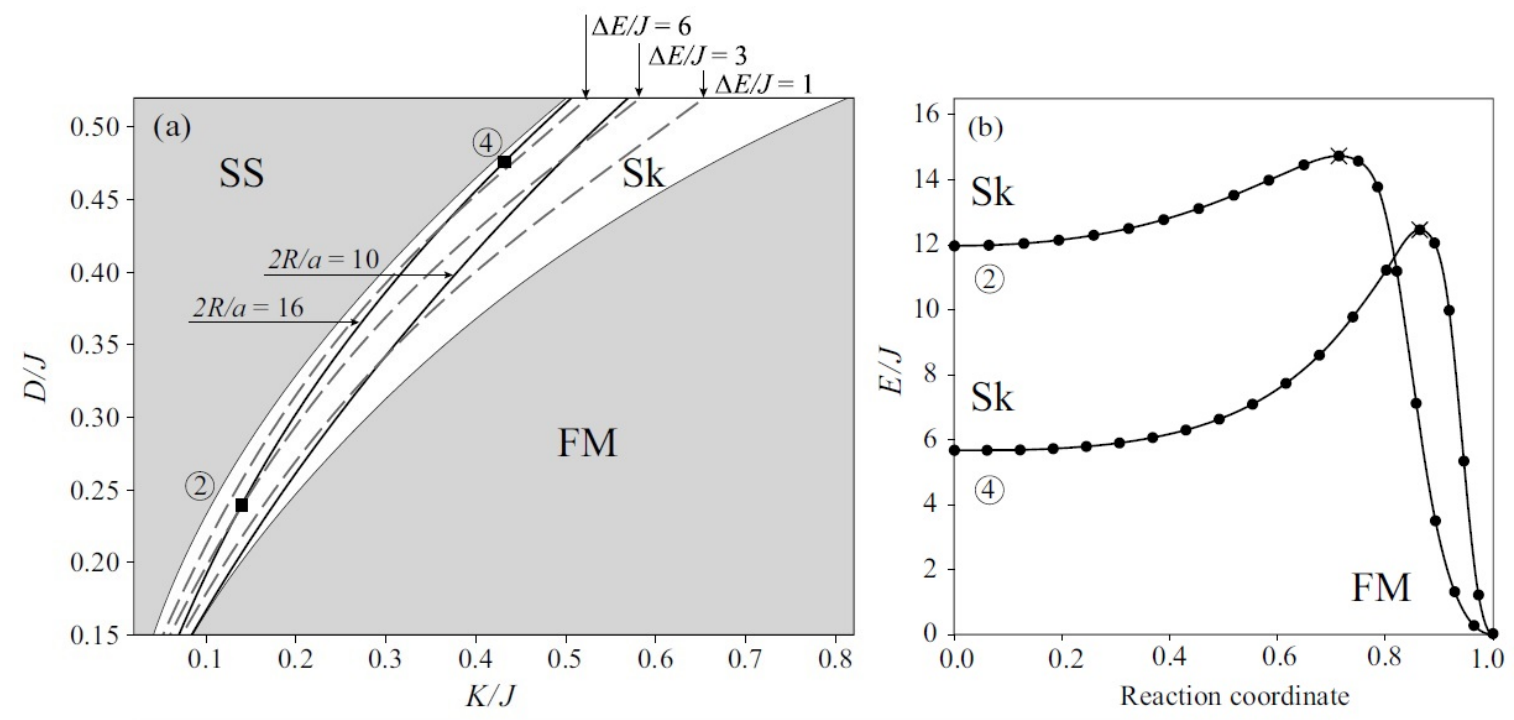

FIG. 2. (a) Zero-temperature phase diagram in reduced variables $K / J$ and $D / J$ for a magnetic monolayer in zero applied magnetic field. Spin spiral is the ground state of the system in the SS region, while the ferromagnetic state is the only stable configuration in the FM region. Isolated skyrmions exist in the Sk sector. Contours of equal skyrmion size are shown with solid lines. Dashed lines represent contours of constant energy barrier for the skyrmion collapse. Two points on the contour where $2 R / a=16$ are labeled with the encircled numbers. For these points, energy variation along the MEP for the skyrmion collapse is shown in the panel (b). The filled circles show position of the intermediate states along the annihilation paths, while crosses indicate energy maxima along the MEPs. The reaction coordinate is defined as the normalized displacement along the MEP. The starting- and end-points of the reaction coordinate are the skyrmion and FM states, respectively

Zero-temperature phase diagram as a function of material parameters $K / J$ and $D / J$ is shown in Fig. 2(a). The diagram is characterized by three regions that correspond to distinct magnetic textures emerging as minima on the energy surface of the system, see Eq. (2). In the SS region, the spin spiral is the ground state of the system. In the FM region, chiral modulations are completely suppressed and polarized ferromagnetic configuration is the only stable configuration. In between these two regions there is an Sk sector where isolated skyrmions exist as metastable states in the FM background [24,25]. The Sk region is separated from the other two by critical lines at which skyrmion solutions become unstable. At the lower critical line, skyrmions spontaneously collapse into the FM state. Observe that the lower line is characterized by vanishing energy barrier rather than vanishing skyrmion size (see Fig. 1). The fact that skyrmions collapse with finite radii is a consequence of the discreteness of the model used here [25,43]. At the upper critical line, isolated skyrmions strip out into spiral states [24,25].

It is informative to trace contours of constant skyrmion diameter and collapse energy barrier in the Sk sector of the magnetic diagram. Here, the following method has been used to obtain the contours. At first, profiles of $R$ and $\Delta E$ as functions of $K / J$ for several values of $D / J$ are calculated. Two profiles for $D / J=24$ and $D / J=28$ are presented in Fig. 1 as an example. For each profile, the value of the anisotropy parameter corresponding to a predefined skyrmion size or energy barrier is isolated by interpolation between the data points, refined using the bisection method and then placed on the $K / J-D / J$ diagram. Finally, the points corresponding to different values of $D / K$ are connected to trace the contour line. Contours of constant $R$ and $\Delta E$ are shown in Fig. 2(a) with solid and dashed lines, respectively. As expected, skyrmions get both larger and more stable against transition to the FM 
state in the vicinity of the strip-out critical line. However, $R$ - and $\Delta E$-isolines do intersect, demonstrating that the skyrmion stability is not a unique function of the skyrmion size. This result is further illustrated by a comparison of the collapse energy barriers for skyrmions of equal size. In particular, two points lying on the same constant $R$ contour are chosen. The points labeled (2) and (4) on the magnetic phase diagram correspond to parameter sets $K / J=0.14, D / J=0.24$ and $K / J=0.44, D / J=0.48$, respectively [see Fig. 2(a)]. The sets result in the same equilibrium skyrmion diameter, $2 R=16 a$, with $a$ being a lattice constant, but different collapse energy barriers, which is clear from Fig. 2(b) showing energy variation along the MEPs for the skyrmion annihilation process.
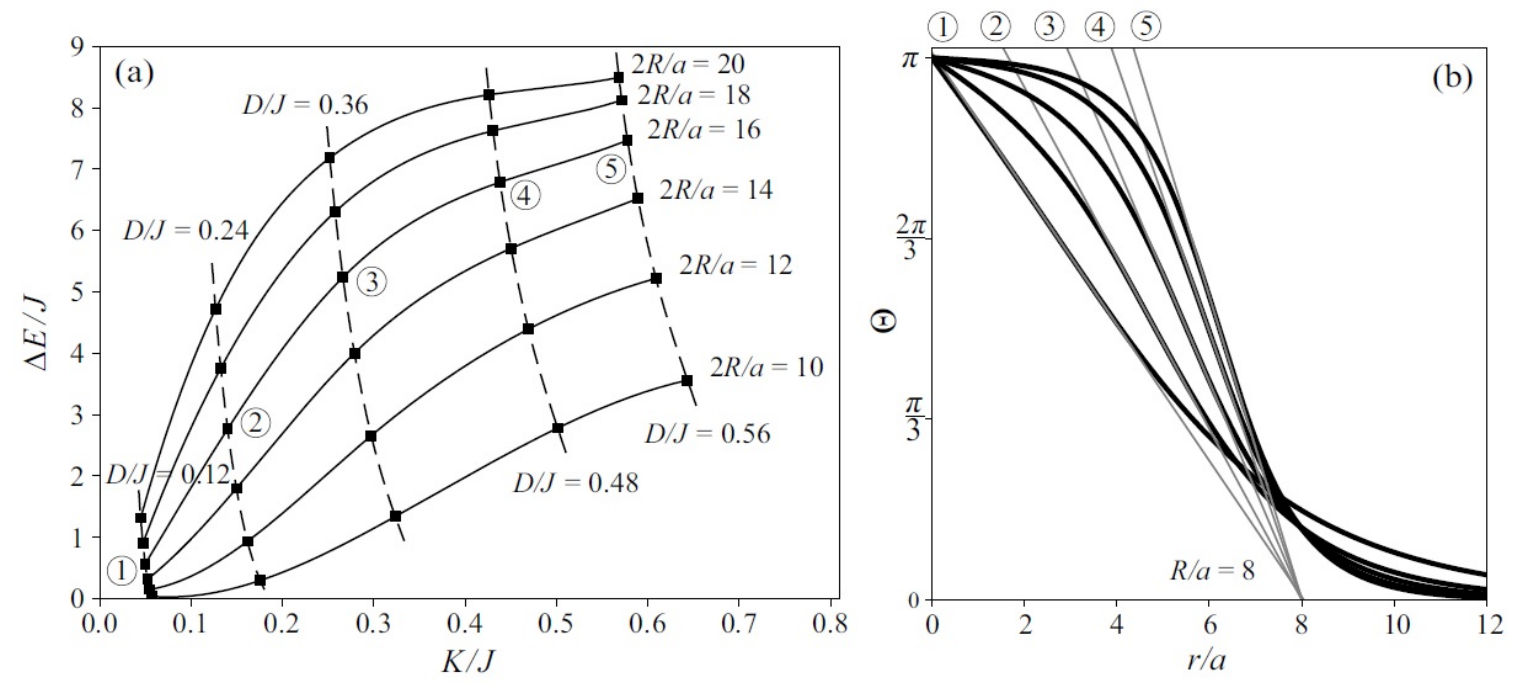

FIG. 3. (a) Energy variation along contours of equal skyrmion size (solid lines). Points corresponding to the same value of the $D / J$ parameter are connected with dashed lines. Encircled numbers label the points for which skyrmion profiles are shown in the panel (b)

How the energy barrier changes along the contours of equal skyrmion size is summarized in Fig. 3(a). For a fixed skyrmion diameter, the collapse barrier gets larger as both $K / J$ and $D / J$ increase in a concerted way. The increase in the energy barrier can be rather pronounced, contributing to the enhancement of skyrmion stability. For example, the barrier for a $16 a$-diameter skyrmion varies by more than an order of magnitude within a chosen range of material parameters [see points labeled (1) - (5) in Fig. 3(a)]. It is important to realize that the barrier enhancement is achieved by a nontrivial parameter transformation rather than simple parameter scaling since reduced values of the anisotropy parameter and DM interaction strength are used. Interestingly, this concerted parameter transformation does not conserve the skyrmion shape, although it does conserve the skyrmion size. The skyrmion profile variation along the contour where $2 R=16 a$ is shown in Fig. 3(b). From Figs. 3(a), 3(b), it is clear that fixed-size skyrmions become more stable as they transform from textures with an arrow-like shape into states that resemble magnetic bubbles where more spins at the core point almost antiparallel $(\Theta \approx \pi)$ to the magnetization of the FM background with $\Theta \approx 0$.

\section{Conclusion}

Our results demonstrate that a change in the shape of fixed-size skyrmions affects the height of the energy barrier protecting the skyrmions from collapse to the FM state. Clearly, enhanced energy barriers for the bubblelike skyrmions should have a direct impact on the skyrmion lifetime. A skyrmion's lifetime at a given temperature can be quantified using the Arrhenius law [see Eq. (1)], but the pre-exponential factor $\tau_{0}$ needs to be evaluated. The prefactor incorporates the entropic and dynamical contributions to the skyrmion's stability. If skyrmion decay induced by thermal fluctuations is a rare event on the intrinsic time scale of the magnetization dynamics of the system, HTST can be used to calculate $\tau_{0}$ [39]. Within the theory, the pre-exponential factor is defined by the curvature of the energy surface at the SP and at the skyrmion state minimum. For the parameter set $K / J=0.05$, $D / J=0.12$ (see point (1) in Fig. 3), the HTST-estimate of $\tau_{0}$ amounts to $7.44 \cdot 10^{2} \tau_{\text {int }}$, with $\tau_{\text {int }}$ being an intrinsic precession time which can be expressed in terms of the on-site magnetic moment $\mu$, exchange interaction parameter $J$ and gyromagnetic ratio $\gamma$ according to the following formula:

$$
\tau_{i n t}=\frac{\mu}{J \gamma} .
$$


Taking $\mu=3$ Bohr magnetons and $J=10 \mathrm{meV}$, which are typical parameter values for two-dimensional magnetic systems based on transition metals [20,29], one obtains a pre-exponential factor of roughly $0.1 \mathrm{~ns}$, a value which is actually often assumed in studies of thermally activated magnetic transitions [32,46,47].

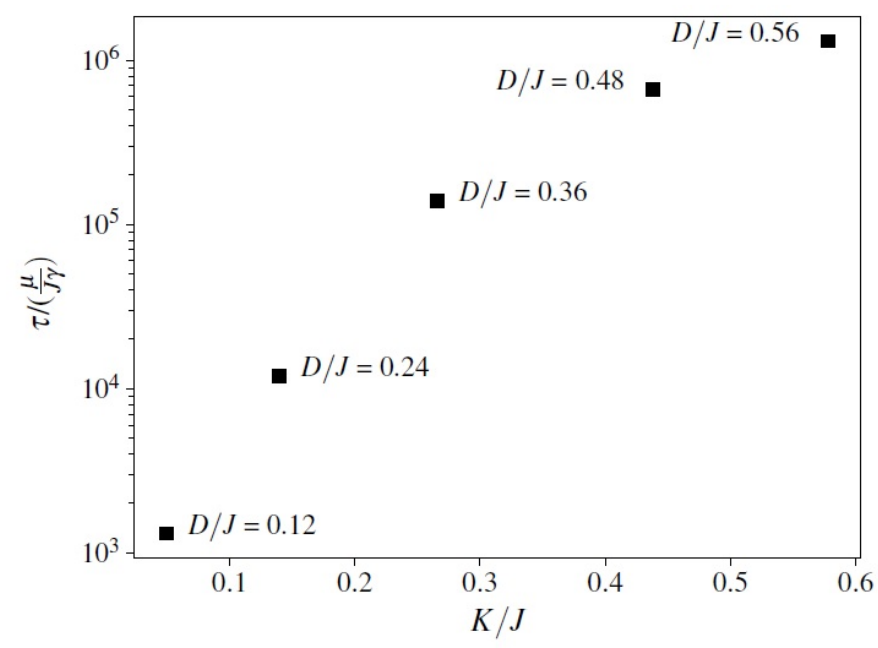

FIG. 4. Calculated skyrmion lifetime along the isoline where $2 R / a=16$. Thermal energy is taken to be $k_{B} T=J$. Constant Arrhenius pre-exponential factor is assumed

Assuming the constant pre-exponential factor and taking thermal energy to be $k_{B} T=J$, Eq. (1) can now be used to calculate the skyrmion lifetime. The calculated results of the lifetime of fixed-size skyrmions $(2 R=16 a)$ as a function of relevant material parameters are presented in Fig. 4. The lifetime increases significantly, by three orders of magnitude within a chosen parameter range. Obtained enhancement of the lifetime is due to the increase in the energy barrier [see Fig. 3(a)] as the constant prefactor approximation is used here. This approximation could, however, be insufficient in some cases. The Arrhenius prefactor can vary by several orders of magnitude depending on the parameters of the magnetic system, as has been demonstrated both experimentally and theoretically for Fe islands on W(110) $[48,49]$. Recent studies suggest that variable pre-exponential factor could be particularly relevant for magnetic skyrmions $[38,50]$. It remains to be seen how the prefactor changes under the variation of material parameters of a skyrmionic system and how this affects the skyrmion lifetime.

In summary, the relationship between the skyrmion size and skyrmion stability has been analyzed based on MEP calculations and atomistic spin Hamiltonian. It has been demonstrated that the energy barrier protecting the skyrmion from collapse to the FM state is not uniquely defined by the skyrmion size, which opens up the possibility to enhance the stability of nanoscale magnetic skyrmions via nontrivial, concerted adjustment of material parameters. The deduced parameter transformation conserves the skyrmion size, but does not conserve the skyrmion shape and is not reduced to a simple parameter scaling. In particular, fixed-size skyrmions transform from textures with an arrow-like shape into states that resemble magnetic bubbles as the anisotropy parameter and the DM interaction strength increase in a concerted way. This transformation of the skyrmion shape is accompanied by an increase in the collapse energy barrier and thus enhancement of skyrmion stability. The prediction that bubble-like skyrmions should be more stable than the arrow-like skyrmions of the same size could help design magnetic materials hosting nanoscale, long-lived skyrmionic bits for future digital technologies.

\section{Acknowledgements}

The authors would like to thank H. Jónsson and V.M. Uzdin for helpful discussions and useful comments. This work is supported by the Russian Science Foundation (Grant No. 17-72-10195).

\section{References}

[1] Bogdanov A. N., Yablonsky D. A. Thermodynamically stable "vortices" in magnetically ordered crystals. The mixed state of magnets. Sov. Phys. JETP., 1989, 68(1), P. 101-103.

[2] Bogdanov A., Hubert A. Thermodynamically stable magnetic vortex states in magnetic crystals. J. Magn. Magn. Mater., 1994, 138(3), P. 255-269.

[3] Bogdanov A., Hubert A. The properties of isolated magnetic vortices. Phys. Stat. Sol. (b), 1994, 186(2), P. 527-543.

[4] Kiselev N.S., Bogdanov A. N., Schäfer R., Rößler U.K. Chiral skyrmions in thin magnetic films: new objects for magnetic storage technologies? J. Phys. D: Appl. Phys., 2011, 44, P. 392001. 
[5] Fert A., Cros V., Sampaio J. Skyrmions on the track. Nat. Nanotechnol., 2013, 8, P. 152-156.

[6] Müller J. Magnetic skyrmions on a two-lane racetrack. New J. Phys., 2017, 19, P. 025002(2017).

[7] Nagaosa N., Tokura Y. Topological properties and dynamics of magnetic skyrmions. Nat. Nanotechnol., 2013 , 8, P. 899-911.

[8] Woo S., Litzius K., Krüger B., Im M.-Y., Caretta L., Richter K., Mann M., Krone A., Reeve R. N., Weigand M., Agrawal P., Lemesh I., Mawass M.-A., Fischer P., Kläui M., Beach G.S.D. Observation of room-temperature magnetic skyrmions and their current-driven dynamics in ultrathin metallic ferromagnets. Nat. Mater, 2016, 15, P. 501-506.

[9] Yu X.Z., Kanazawa N., Zhang W.Z., Nagai T., Hara T., Kimoto K., Matsui Y., Onose Y., Tokura Y. Skyrmion flow near room temperature in an ultralow current density. Nat. Commun., 2012, 3, P. 988.

[10] Sampaio J., Cros V., Rohart S., Thiaville A., Fert A. Nucleation, stability and current-induced motion of isolated magnetic skyrmions in nanostructures. Nat. Nanotechnol., 2013, 8, P. 839-844.

[11] Moreau-Luchaire C., Moutas C., Reyren N., Sampaio J., Vaz C. A. F., Van Horne N., Bouzehouane K., Garcia K., Deranlot C., Warnicke P., Wohlhüter P., George J.-M., Weigand M., Raabe J., Cros V., Fert A. Additive interfacial chiral interaction in multilayers for stabilization of small individual skyrmions at room temperature. Nat. Nanotechnol., 2016, 11, P. 444-448.

[12] Boulle O., Vogel J., Yang H., Pizzini S., de Souza Chaves D., Locatelli A., Menteş T. O., Sala A., Buda-Prejbeanu L. D., Klein O., Belmeguenai M., Roussigné Y., Stashkevich A., Chérif S. M., Aballe L., Foerster M., Chshiev M., Auffret S., Miron I. M., Gaudin G. Room-temperature chiral magnetic skyrmions in ultrathin magnetic nanostructures. Nat. Nanotechnol., 2016, 11, P. $449-454$.

[13] Yu G., Upadhyaya P., Li X., Li W., Kim S. K., Fan Y., Wong K. L., Tserkovnyak Y., Amiri P. K., Wang K. L. Room-temperature creation and spinorbit torque manipulation of skyrmions in thin films with engineered asymmetry. Nano Lett., 2016, 16(3), P. 1981-1988.

[14] Jiang W., Upadhyaya P., Zhang W., Yu G., Jungfleisch M. B., Fradin F. Y., Pearson J. E., Tserkovnyak Y., Wang K. L., Heinonen O., te Velthuis S. G. E., Hoffmann A. Blowing magnetic skyrmion bubbles. Science, 2015, 17(6245), P. $283-286$.

[15] Legrand W., Maccariello D., Reyren N., Garcia K., Moutafis C., Moreau-Luchaire C., Collin S., Bouzehouane K., Cros V., Fert A. Room-temperature current-induced generation and motion of sub-100 nm skyrmions. Nano Lett., 2017, 17(4), P. 2703-2712.

[16] Jiang W., Zhang X., Yu G., Zhang W., Wang X., Jungfleisch M. B., Pearson J.E., Cheng X., Heinonen O., Wang K. L., Zhou Y., Hoffmann A., te Velthuis S. G. E. Direct observation of the skyrmion Hall effect. Nat. Phys., 2016, 11, P. $449-454$.

[17] Hrabec A., Sampaio J., Belmeguenai M., Gross I., Weil R., Chérif S. M., Stashkevich A., Jacques V., Thiaville A., Rohart S. Currentinduced skyrmion generation and dynamics in symmetric bilayers. Nat. Commun., 2017, 8, P. 15765.

[18] Litzius K., Lemesh I., Krüger B., Bassirian P., Caretta L., Richter K., Büttner F., Sato K., Tretiakov O. A., Förster J., Reeve R. M., Weigand M., Bykova I., Stoll H., Schütz G., Beach G.S.D., Kläui M. Skyrmion Hall effect revealed by direct time-resolved X-ray microscopy. Nat. Phys., 2017, 13, P. 170-175.

[19] Romming N., Hanneken C., Menzel M., Bickel J. E., Wolter B., von Bergmann K., Kubetzka A., Wiesendanger R. Writing and deleting single magnetic skyrmions. Science, 2013, 341(6146), P. 636-639.

[20] Romming N., Kubetzka A., Hanneken C., von Bergmann K., Wiesendanger R. Field-dependent size and shape of single magnetic skyrmions. Phys. Rev. Lett., 2015, 114(17), P. 177203.

[21] Hanneken C., Otte F., Kubetzka A., Dupé B., Romming N., von Bergmann K., Wiesendanger R., Heinze S. Electrical detection of magnetic skyrmions by tunnelling non-collinear magnetoresistance. Nat. Nanotechnol., 2015, 10, P. 1039-1042.

[22] Rößler U.K., Leonov A. A., Bogdanov A. N. Chiral skyrmionic matter in non-centrosymmetric magnets. J. Phys. Conf. Ser., 2011, 303, P. 012105.

[23] Rößler U. K., Bogdanov A. N., Pfleiderer C. Spontaneous skyrmion ground states in magnetic metals. Nature, 2006 , 442, P. 797-801.

[24] Wilson M.N., Butenko A.B., Bogdanov A.N., Monchesky T. L. Chiral skyrmions in cubic helimagnet films: The role of uniaxial anisotropy. Phys. Rev. B, 2014, 89, P. 094411.

[25] Leonov A. O., Monchesky T. L., Romming N., Kubetzka A., Bogdanov A. N., Wiesendanger R. The properties of isolated chiral skyrmions in thin magnetic films. New J. Phys., 2016, 18, P. 065003.

[26] Bogdanov A., Hubert A. The stability of vortex-like structures in uniaxial ferromagnets. J. Magn. Magn. Mater., 1999, 195(1), P. 182-192.

[27] Bessarab P. F., Uzdin V. M., Jónsson H. Method for finding mechanism and activation energy of magnetic transitions, applied to skyrmion and antivortex annihilation. Comput. Phys. Commun., 2015, 196, P. 335-347.

[28] Rózsa L., Simon E., Palotás K., Udvardi L., Szunyogh L. Complex magnetic phase diagram and skyrmion lifetime in an ultrathin film from atomistic simulations. Phys. Rev. B, 2016, 93, P. 024417.

[29] Rohart S., Miltat J., Thiaville A. Path to collapse for an isolated Néel skyrmion. Phys. Rev. B, 2016, 93, P. 214412.

[30] Hagemeister J., Romming N., von Bergmann K., Vedmedenko E. Y., Wiesendanger R. Stability of single skyrmionic bits. Nat. Commun., 2015,6 , P. 8455.

[31] Lobanov I. S., Jónsson H., Uzdin V. M. Mechanism and activation energy of magnetic skyrmion annihilation obtained from minimum energy path calculations. Phys. Rev. B, 2016, 94, P. 174418.

[32] Stosic D., Mulkers J., Van Waeyenberge B., Ludermir T., Milošević M. V. Paths to collapse for isolated skyrmions in few-monolayer ferromagnetic films. Phys. Rev. B, 2017, 95, P. 214418.

[33] Uzdin V.M., Potkina M. N., Lobanov I. S., Bessarab P. F., Jónsson H. The effect of confinement and defects on the thermal stability of skyrmions. Physica B: Condens. Matter. In press. DOI: 10.1016/j.physb.2017.09.040.

[34] Cortés-Ortuño D., Wang W., Beg M., Pepper R. A., Bisotti M.-A., Carey R., Vousden M., Kluyver T., Hovorka O., Fangohr H. Thermal stability and topological protection of skyrmions in nanotracks. Sci. Rep., 2017, 7, P. 4060.

[35] Uzdin V. M., Potkina M. N., Lobanov I. S., Bessarab P. F., Jónsson H. Energy surface and lifetime of magnetic skyrmions. J. Magn. Magn. Mater., 2018, 459, P. 236-240.

[36] Stosic D., Ludermir T. B., Milošević M.V. Pinning of magnetic skyrmions in a monolayer Co film on Pt(111): Theoretical characterization and exemplified utilization. Phys. Rev. B, 2017, 96, P 214403.

[37] von Malottki S., Dupé B., Bessarab P. F., Delin A., Heinze S. Enhanced skyrmion stability due to exchange frustration. Sci. Rep., 2017, 7, P. 12299.

[38] Bessarab P.F., Müller G.P., Lobanov I.S., Rybakov F. N., Kiselev N.S., Jónsson H., Uzdin V. M., Blügel S., Bergqvist L., Delin A. Lifetime of racetrack skyrmions. Sci. Rep., 2018, 8, P. 3433.

[39] Bessarab P. F., Uzdin V. M., Jónsson H. Harmonic transition-state theory of thermal spin transitions. Phys. Rev. B, 2012, 85, P. 184409. 
[40] Draaisma H. J.G., de Jonge W. J. M. Surface and volume anisotropy from dipole-dipole interactions in ultrathin ferromagnetic films. J. Appl. Phys., 1988, 64(7), P. 3610-3613.

[41] Bessarab P. F. Comment on "Path to collapse for an isolated Néel skyrmion". Phys. Rev. B, 2017,95, P. 136401.

[42] Butenko A. B., Leonov A. A., Bogdanov A. N., Rößler U. K. Theory of vortex states in magnetic nanodisks with induced DzyaloshinskiiMoriya interactions. Phys. Rev. B, 2009, 80, P. 134410.

[43] Siemens A., Zhang Y., Hagemeister J., Vedmedenko E. Y., Wiesendanger R. Minimal radius of magnetic skyrmions: statics and dynamics. New J. Phys., 2016, 18, P. 045021.

[44] Büttner F., Lemesh I., Beach G. S. D. Theory of isolated magnetic skyrmions: From fundamentals to room temperature applications. Sci. Rep. 2018. 8, P. 4464.

[45] Rohart S., Thiaville A. Skyrmion confinement in ultrathin film nanostructures in the presence of Dzyaloshinskii-Moriya interaction. Phys. Rev. B, 2013, 88, P. 184422.

[46] Bedanta S., Kleemann W. Supermagnetism. J. Phys. D, 2008, 42, P. 013001.

[47] Kapaklis V., Arnalds U.B., Farhan A., Chopdekar R. V., Balan A., Scholl A., Heyderman L. J., Hjörvarsson B. Thermal fluctuations in artificial spin ice. Nat. Nanotechnol., 2014, 9, P. 514-519.

[48] Krause S., Herzog G., Stapelfeldt T., Berbil-Bautista L., Bode M., Vedmedenko E. Y., Wiesendanger R. Magnetization reversal of nanoscale islands: How size and shape affect the Arrhenius prefactor. Phys. Rev. Lett., 2009, 103, P. 127202.

[49] Bessarab P. F., Uzdin V. M., Jónsson H. Size and shape dependence of thermal spin transitions in nanoislands. Phys. Rev. Lett., 2013, 110, P. 020604.

[50] Wild J., Meier T. N. G., Pöllath S., Kronseder M., Bauer A., Chacon A., Halder M., Schowalter M., Rosenauer A., Zweck J., Müller J., Rosch A., Pfleiderer C., Back C. H. Entropy-limited topological protection of skyrmions. Sci. Adv., 2017, 3(9), P. e1701704. 